Simulating Collective Behavior in the

Movement of Immigrants by Using a Spatial Prisoner's Dilemma with Move Option

Bingchen LIN, Can ZHOU, Zhe HUA, Guangdi HU, Ruxin DING, Qiyuan ZENG, and Jiawei LI

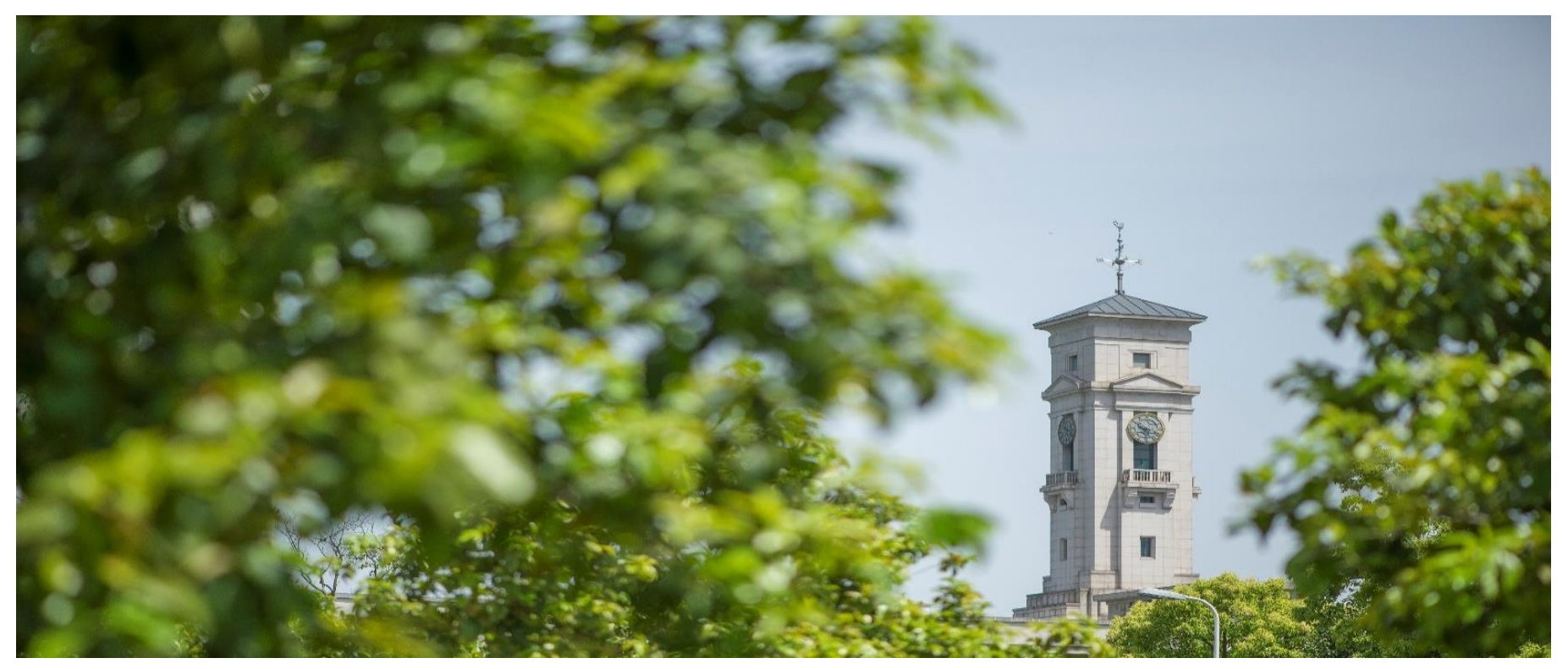


Faculty of Science and Engineering, University of Nottingham Ningbo China, 199 Taikang East Road, Ningbo, 315100, Zhejiang, China.

First published 2020

This work is made available under the terms of the Creative Commons Attribution 4.0 International License:

http://creativecommons.org/licenses/by/4.0

The work is licenced to the University of Nottingham Ningbo China under the Global University Publication Licence:

https://www.nottingham.edu.cn/en/library/documents/researchsupport/global-university-publications-licence.pdf 


\title{
Simulating Collective Behavior in the Movement of Immigrants by Using a Spatial Prisoner's Dilemma with Move Option
}

\author{
Bingchen LIN*, Can ZHOU*, Zhe HUA, Guangdi HU, Ruxin DING, Qiyuan \\ ZENG, and Jiawei LI凶 \\ University of Nottingham Ningbo China \\ Email: jiawei.li@nottingham.edu.cn
}

\begin{abstract}
The movement of immigrants is simulated by using a spatial Prisoner's Dilemma (PD) with move option. We explore the effect of collective behavior in an evolutionary migrating dynamics. Simulation results show that immigrants adopting collective strategy perform better and thus gain higher survival rate than those not. This research suggests that the clustering of immigrants promotes cooperation.
\end{abstract}

Keywords: simulation · collective behavior · spatial Prisoner's Dilemma - evolutionary game theory

\section{Introduction}

Competition and cooperation are two ubiquitous and inalienable actions in nature [20]. How cooperation evolves in a population of self-interest individuals is an important longstanding scientific question in both biology and social sciences. A powerful framework which has been used frequently to investigate these problems is evolutionary game theory, which includes the changing strategy adoption of a single agent, leading to the concept of rounds [19].

The most relevant game models are two-player games where two players choose to either cooperate $(C)$ or defect $(D)$. The payoff of a player depends on the choices of both sides. There are four possible payoffs in total. Both two players will get a reward $(R)$ if they both choose to cooperate while a punishment $(P)$ will be given if they both choose to defect. In the situation where one player chooses to cooperate whereas the other player chooses to defect. A temptation reward $(T)$ will be given to the defector, whereas the cooperator will get the suckers punishment $(S)$. Different ranking about the above four values determines what game they are playing. In PD game, four values are set as $T>R>P>S$. In terms of these inequalities, it is clear that defection is dominant: every player is better off to choose defect whatever the other player chooses. This makes mutual defection to be a stable strategy in a well-mixed population. There are two other relevant social dilemma games with PD [10]. The first game is called Chicken [15]

\footnotetext{
* These authors contributed equally to this work
} 
or Snowdrift (SD) with a rank as $T>R>S>P$. A reasonable explanation is that in most animal contests, mutual defection will lead to the worst consequence for both players where the loss even exceeds the cost of being exploited. Another game is called Stag Hunt (SH) [3]. The rank set in this game is $R>T>P>S$, which means the reward of cooperation surpasses the temptation. An example of this situation is that when disasters or powerful enemies occur, it is meaningless to still defect other self-interested agents. In the next part of the introduction, evolutionary games with spatial structure will be discussed.

Unlike classical evolutionary game theory which considers the population of players as randomly mixed, a spatial game model puts all players into a two-dimensional lattice, allowing them to continuously interact with their direct neighbors. This spatial structure simulates strategic interactions in the real world, where individuals are placed in certain locations, not move far away from their birthplace and only interact with their relatively fixed neighbors [8]. In each generation of a spatial PD game, every player will play a series of twoplayer PD games with all its neighbors (Moore neighborhood is commonly used here, which indicates the players in eight direct-linked sites) and accumulate the payoff. At the end of each generation, each player will compare its payoff with all its neighbors and change its strategy, following the one with the highest payoff among them. Compared with the classical evolutionary game, the competition in the spatial game is more complex, and it may lead to different but more useful results. While defection dominates cooperation in PD game, the existence of spatial structure gives cooperation the possibility of survival and grow into clusters $[13,22]$. Inside a cluster, the cooperation behavior provides its members with enough payoff to overweight the exploitation from outside defectors [23]. In a scale-free model where strong correlations exist between individuals, cooperation may even dominate over defection [18].

In 2007, Vainstein et al. introduced a random-walk mechanism to spatial game model, in which each player can move to a randomly chosen empty neighboring site with a probability [23]. Later, more directional migration mechanisms were introduced. Meloni et al. studied the case where the direction of migration is payoff biased and the movement is with a certain velocity [12]. Helbing et al. introduced the famous success-driven migration model, where players move to the empty neighboring site with the highest estimated payoff. In neighbor-considered migration model [16], a new concept called fairness payoff is introduced. In this model, while players still tend to move to the sites with a higher payoff, reducing the disparity of payoff among their neighbors becomes another target.

To date, there are many studies focused on the effects of mobility on the evolution of cooperation of two or more groups(strategies) in a spatial version of the N-player PD game. However, group cooperation in humans and animals is common $[3,21]$, collective strategies also have great potential research value.

The collective strategy is a summary or a systematic name of collective behaviors. The concept of collective behavior traced back to Park and Burgess [14] and Blumer [9]. It is a special kind of social interaction and refers to a group of unorganized people who are stimulated and influenced by a certain factor. This 
expression has been expanded to other creatures, such as cells, social animals like birds and fish, and insects including ants [7].

Immigration is the international migration of people into a destination country in order to settle or reside there. Typical immigrants include permanent residents, naturalized citizens or those who expect to be employed as a temporary foreign worker or as a migrant worker [11]. Immense studies about influences brought by immigrants has been done. In terms of economic effects, research suggests that both sending and receiving countries benefit from migration $[6$, 1]. Furthermore, some researches drew a conclusion that immigration on average has positive economic influences towards the native population. However, it is unclear whether low-skilled immigrants have negative effects on low-skilled natives $[4,2]$. In conclusion, immigration is a ubiquitous phenomenon in human societies, and it influences many aspects of social development. Therefore, immigration contains a significant value to be studied and immigration model is a useful one chosen by many other researches. Collective behavior brings advantage to the group of players who implements it. However, the performance of collective strategy on immigrants still needs more studies. Therefore, this paper studies if collective behavior is still powerful and how powerful it is for immigrants to survive in a new environment.

Though there has been a number of studies about migration and collective strategy separately, there are few studies combine migration with collective behavior. In this paper, we will continue this procession of research by studying the performance of collective strategies as immigrants in a spatial N-player PD game with random mobility.

\section{Model: a Spatial Prisoners Dilemma (SPD) with Move Option}

In the game model of this paper, some modification is done to the migration method in order to emphasize the existence of strategic interaction. The migration method is similar with that in Schelling model, which is a long studied mathematical model in social sciences. In Schelling model [17], a map is initialized with randomly distributed agents from several groups where each group represents a race. Each agent has an indicator of satisfaction, which value will increase as the number of its neighbors of the same race increases. Agents are stimulated to move if they are unsatisfied with their own satisfaction level. The studies on Schelling model came out with that even a slight homophyllic bias will lead to significant segregation of the different groups of agents. Since parts of the Schelling model corresponds to the spatial migrating PD game (for example, the spatial structure, the interaction with the neighbors, and the migrating behavior), some ideas from Schelling model is applied into our game model. More specifically, similar with Schelling model, in our game model player with collective strategy will stop moving only if the number of its neighbors of same group is equal or larger than a predefined mobility. 
The game model introduced in this paper divides the player into two groups, natives and immigrants. The numbers of players in the two groups are not equal, while the population of natives is at least ten times more than the population of immigrants. The tag of the two groups is visible to all players. The players strategy may depend on its opponents group. According to Chiong and Kirley [5] , proper move action can enhance the cooperation between immigrants when compared with the natives who always stay at the same place. In this paper, the effect of collective behaviour will be observed with movement, which might come to a more conspicuous and observable result. In the game model, immigrants with collective strategy will invade a larger group of native inhabitants. The collective behavior is reflected in two aspects. Firstly, the player with collective strategy could identify its opponents identity; it will always cooperate with the player from the same group and defect with the player from different groups. Secondly, the player with collective strategy tends to settle down in environments where its group dominates. A real-world example of this game model is the immigration in human society, where the native people is hostile toward the immigrant. To survive in the new unfriendly environment, isolated immigrants may assemble and develop as a group. Through this study, a new game model about collective behavior and migration is generated, which may fill some vacancy in the existing studies.

In this section, three perspectives will be introduced and expounded: the static rule of the game, the method under this set of rules and the experiment settings. Key terms are explained below:

group a collection of all players using the same strategy.

native a group having a larger quantity and have a low tendency of movement. immigrant a group having a less quantity and have a comparably higher tendency of movement.

neighbor a player located near the current player. A player can have at most eight neighbors (Moore Neighborhood).

mobility a value indicating tendency of movement of a collective strategy, in this paper, referring to the minimum number of neighbors of same strategy in neighborhood to activate movement. Instances of strategies with different mobility can be seen in Appendix.

\section{$2.1 \quad$ Rule}

This model implements a spatial PD game; therefore, previous traditional studies are referenced for the rules.

- Every player locates in a cell of the 2D map.

- There are $50 \%$ players on the original map.

- Two adjacent players play a single PD game in an iteration.

- The result of each PD game is a pair of payoff value adding to scores of two players. The score is determined according to the payoff matrix in Fig. 1. After playing with each neighbor, the score (total payoff in this iteration) of 
central player will be determined. At the start of each iteration, scores of all players will be reset to 0 .

- The game ends when no change can be made or one of the two groups disappears.

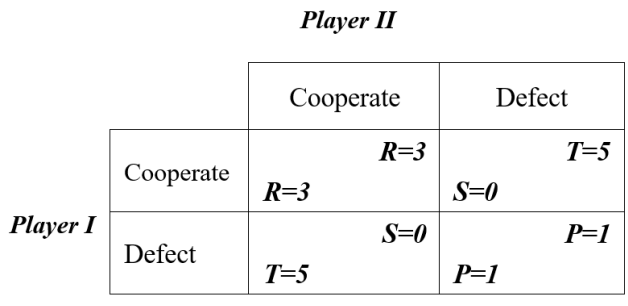

Fig. 1. The payoff matrix

In this game, several rules are set in order to evaluate and simulate the relationship transition of two groups.

Players are allowed to move to a random nearby empty cell. The move decision is generated based on strategies.

At the end of every iteration, a player adopts the strategy of the neighbour who has received the highest score. If two neighbours gain equal highest score, one strategy will be chosen randomly.

The group adopts an efficient strategy dominates other groups with weak strategy over time.

Therefore, efficiency and strength of each strategy can be estimated according to the number of players remained in evolution.

Six strategies are tested in simulations. Strategies for native players include AllD, AllC and CS. Strategies for immigrants are AllDM, AllCM and CSM. Details of these strategies are given in the Appendix. Different distribution of initial population leads to different result which could be an equilibrium state where native strategies and immigrant strategies coexist, or a homogeneous population In this game, the natives are supposed to stay at the location they are and the immigrants choose whether to move based on their payoffs. In detail, AllD shows the exclusiveness of the native and AllC shows the hospitality of the native. In addition, CS probably dominate in most PD competitions that allows players to cooperate with kin members and defects against non-kin members. This is a strong simulation of group behaviour, which reflects the characteristic of immigrants as well. Besides, AllDM, AllCM and CSM are the same as the three strategies above, except the movement.

To simulate the typical scenarios in the movement of immigrants, it is distinct that immigrants represent less part of the population comparing to the native. In addition, it is possible to develop a different ratio of natives and immigrants. 
Therefore, $20 \%$ and $10 \%$ are applied in the study to restore society. The reason for implementing different ratio is to observe the influence of the number of players on the survival rate in spatial PD. Accordingly, a more convincing conclusion could be drawn even though with a different ratio of two groups.

In our first series of experiments, two percentages of immigrants are simulated: $20 \%$ and $10 \%$. In those experiments, different ratios of players and different strategies are adopted. There are 18 experiments in total. Different strategy combinations are listed below.

1. Native strategy is AllD, immigrant strategies are AllDM, AllCM and CSM respectively.

2. Native strategy is AllC, immigrant strategies are AllDM, AllCM and CSM respectively.

3. Native strategy is CS, immigrant strategies are AllDM, AllCM and CSM respectively.

In another series of experiments, different mobility levels of CSM are tested.

1. Native strategy is AllD, immigrant strategies are CSM, CSM2, CSM3 and CSM4 respectively.

2. Native strategy is AllC, immigrant strategies are CSM, CSM2, CSM3 and CSM4 respectively.

3. Native strategy is CS, immigrant strategies are CSM, CSM2, CSM3 and CSM4 respectively.

\section{SIMULATION RESULTS ANALYSIS}

In the first series of experiments, we focus on whether collective strategy helps immigrants survive among natives. Firstly, three groups of tests were made, in each group the same native strategy played against three immigrant strategies ALLCM, ALLDM and CSM in separated simulations.

Fig. 2 shows that CSM strategy could survive in the environments full of one of the three strategies (ALLD, ALLC, CS). In Fig. 2(a), where the native inhabitants take ALLD strategy, CSM not only becomes the only survival immigrant strategy, but also reaches a high percentage in the population (about $94 \%$ ). In Fig. 2(b), among natives of ALLC strategy, the increasing rate of CSM is as fast as ALLDM's. The difference between results of CSM and ALLDM is that, while the immigrants with ALLDM strategy take the place of all native inhabitants, the percentage of CSM strategy increased to about $90 \%$ and reached an equilibrium. In the games of Fig. 2(c), the native inhabitants with collective strategy is a threat to all immigrant strategies. At the initial iterations, the percentages of population of all immigrant strategies plummeted. However, the immigrants with ALLDM strategy has a high rate may disappear during the plummet stage. The CSM and ALLCM immigrants have a high probability of maintaining a low population and surviving through the game. 


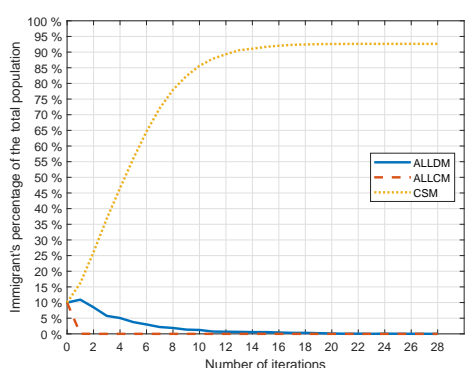

(a) Native strategy: ALLD

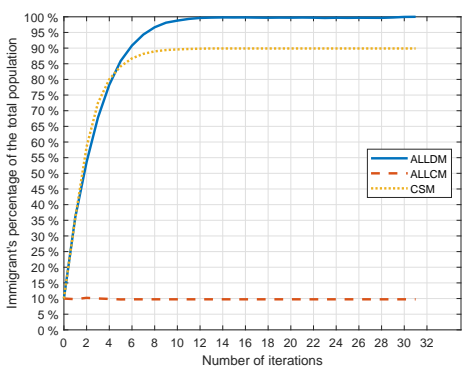

(b) Native strategy: ALLC

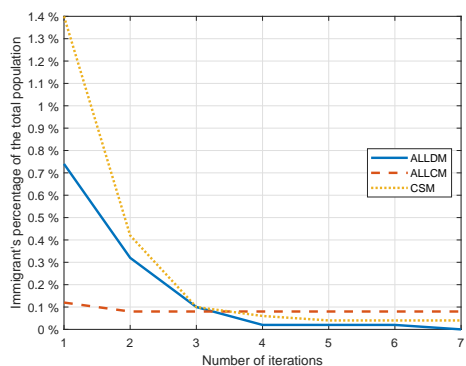

(c) Native strategy: CS

Fig. 2. Changes in immigrant population with the initial population containing $10 \%$ immigrants.

To see if the initial percentage of immigrants will affect the process of game, another group of games are played (the result is shown in Fig. 3), where the initial percentage of immigrant increased from $10 \%$ to $20 \%$. The trends of data in Fig. 3 is generally same as that in Fig. 2, which indicates that the change of initial population not significantly affect the process of game.

Through these two groups of games, three discoveries are drawn. Firstly, while ALLDM and ALLCM both failed to survive in all games, CSM successfully survived in all games. Though in some games, CSM could only maintain a minimal subsistence, in majority of the games CSM led the immigrants to prosperity. In summary, CSM has proven its ability of surviving in SPD games with migration. Collective strategy is still in advantage in this SPD game simulating migration.

The second discovery is that, under most situations, though CSM could rapidly reach a high population, it will get into equilibrium at that population, instead of totally take place of the native inhabitants. This phenomenon was especially significant in Fig. 2(b) and Fig. 3(b), where the ALLDM soon deracinated the native inhabitants, CSM still left some living space for the native inhabitants. Though ALLDM and CSM both take defection strategy toward the native inhabitants, the invasion of CSM is more moderate than ALLDM. A probable reason for this phenomenon is that at the later iterations of the game the CSM 
immigrants has reached a big population and settled down among clusters of immigrants. According to CSM strategy, when CSM immigrants have neighbors from the same group, they will not move around anymore. As the population and density of CSM increases, the movability of CSM immigrants will decrease. After the CSM immigrants formed a stable cluster, they had no more motivation to move across some empty cells to invade the residual native inhabitants.

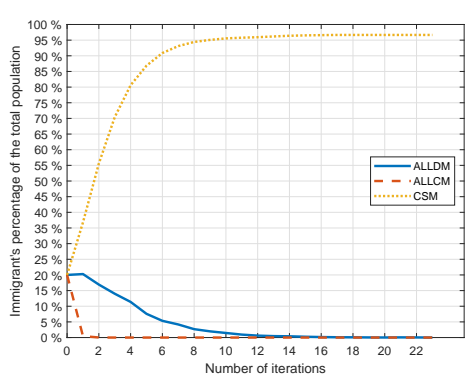

(a) Native strategy is ALLD

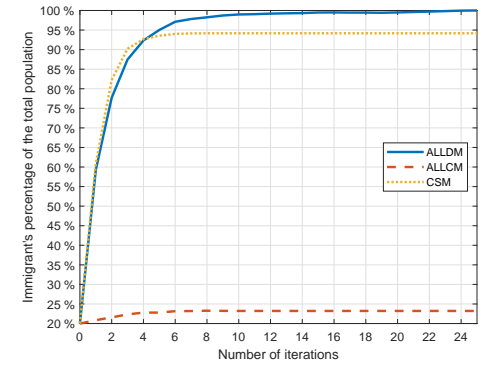

(b) Native strategy is ALLC

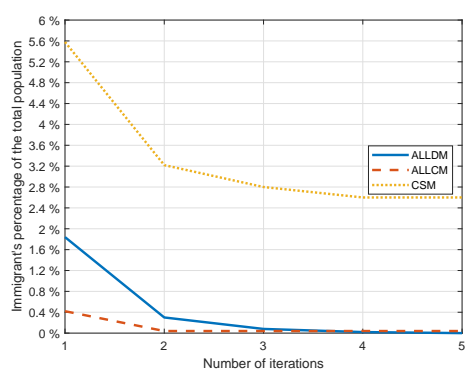

(c) Native strategy is CS

Fig. 3. Changes in immigrant population with the initial population containing $20 \%$ immigrants.

Thirdly, as Fig. 4(d) shows, the empty spaces between native inhabitants and immigrants will segregate the two groups, preventing the residual native inhabitants from extinction. The small cluster of native inhabitants will not be invaded by immigrants, so the native inhabitants could still survive in an environment where CSM immigrants prospered. 


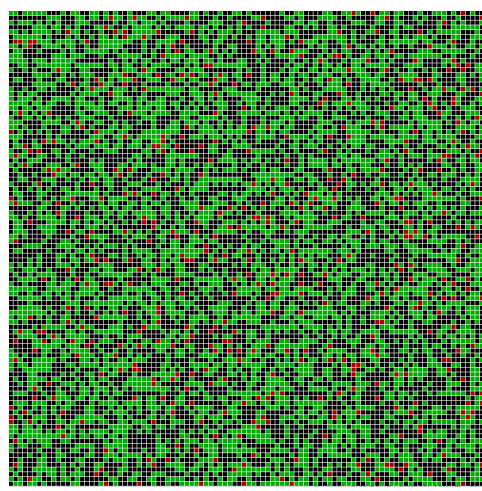

(a) Initial population

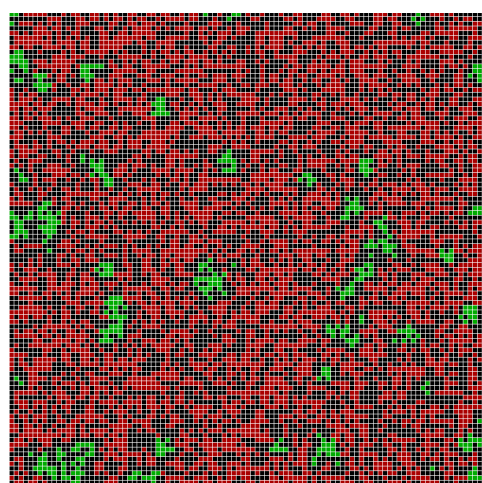

(c) Population in equilibrium

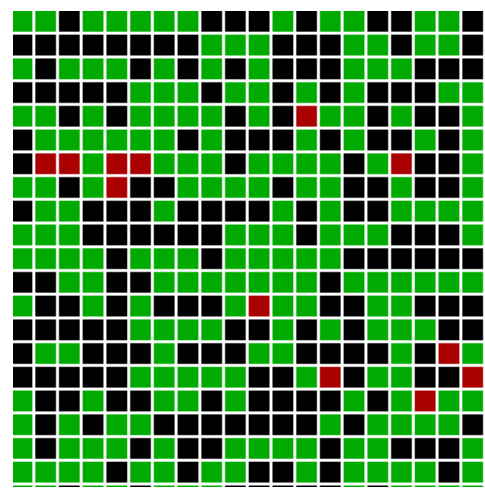

(b) A part of initial population

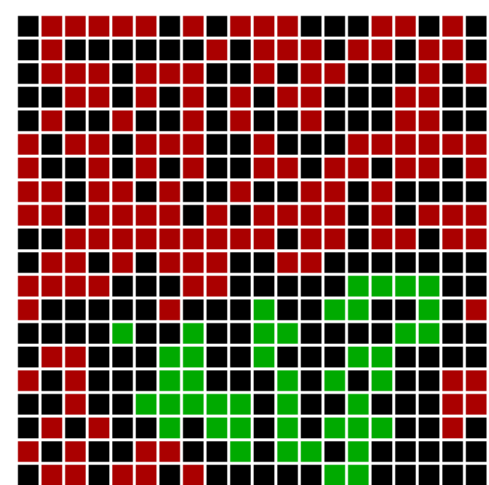

(d) A part of final population

Fig. 4. Simulating migration process. Green (light) cells are natives with ALLC strategy. Red (dark) cells are CSM immigrants. Black cells are empty spaces. The initial population contains $10 \%$ immigrants and $90 \%$ natives.

Moreover, there is a hypothesis that the move condition may affect the experimental results in CSM. As shown in Fig. 5, CSM with different move conditions are compared at the same initial ratio of immigrants. The move conditions have a limited impact on the final result. They influence the final ratio of immigrants in equilibrium population. However, they do not determine whether an immigrant strategy survive or not.

There are four kinds of move conditions for CS, which are when the mobility of move is $1,2,3$ and 4 . The higher the mobility means the higher probability of move.

In the case where natives are ALLD (Fig. 5(a)) and ALLC (Fig. 5(b)), all CSM immigrants will eventually become dominant populations. From the overall trend, the higher the mobility, the greater the probability of movement, and the higher the percentage of immigrant in the total population at equilibrium. It is 
worth noting that when the mobility is 4 -the most active one, the immigrant has a great probability of accounting for $100 \%$ of the total population.

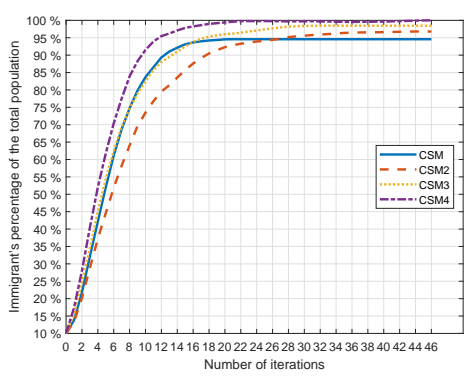

(a) Native strategy is ALLD

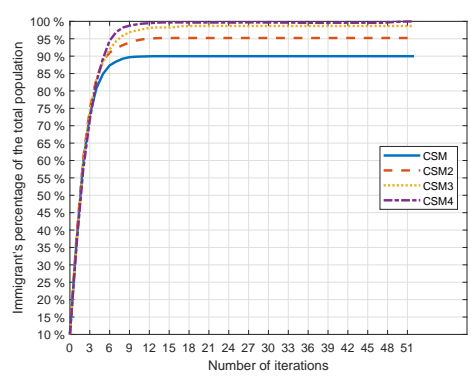

(b) Native strategy is ALLC

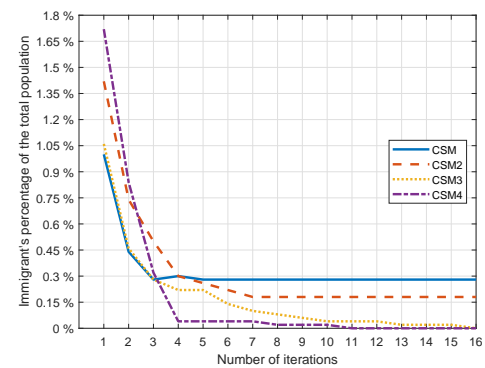

(c) Native strategy is CS

Fig. 5. Changes in immigrant population.

When the native strategy is CS and the immigrant strategy is CSM (Fig. $5(\mathrm{c})$ ), the survival rate of immigrant is sharply reduced. With the increase in mobility, the proportion of immigrant to the total population becomes lower at equilibrium. When the mobility is the highest (4), the immigrant has a high probability of dying.

To sum up, this experiment compares several collective strategies as the survivability of immigrant. When natives use ALLD or ALLC strategy, the higher the mobility, the better the growth rate and the higher the final population ratio. However, in the unfavourable case - the natives use CS strategy, when the mobility grows, the rate of population reduction is higher, and the final proportion of the population is lower, even extinct.

\section{Conclusion}

This paper has discussed the effects of collective strategy in spatial PD via two series of experiments. We have analysed different results when adopting 
diverse strategies. The investigation shows that, comparing with immigrants without collective behaviour, those with collective strategy have better survival rate and a higher proportion of population in equilibrium. We have also studied collective behaviours with different levels of mobility. The result suggests that the mobility of CS has an influence on the survival rate of immigrants. AllC and AllD strategy of the native tends to accelerate the growth rate of immigrants, while the population of immigrants declines and even extinct when the native strategy is CS.

Although the current study is based on a small sample of experiments, it shows the significance of collective behaviour in strategic interactions. Different settings of the game may have an influence on the final result. For example, the initial population are set to $50 \%$ of the lattice, which might lead to a different result if the value is changed. Further research will be conducted in the future with different settings.

\section{Appendix}

Always Defect (AllD) Defect on every move.

Always Cooperate (AllC) Cooperate on every move.

Collective Strategy (CS) Cooperate with kin members and defect against non-kin members.

Always Cooperate with Move (AllCM) Cooperate on every move. If there exist kin members in neighborhood, then stay, else randomly move to one of eight neighboring empty places.

Always Defect with Move (AllDM) Defect on every move. If there exist kin members in neighborhood, then stay, else randomly move to one of eight neighboring empty places.

Collective Strategy with Move (CSM) Cooperate with kin members and defect against non-kin members. If there exist kin members in neighborhood, then stay, else randomly move to one of eight neighboring empty places.

Collective Strategy with Move (CSM2) Cooperate with kin members and defect against non-kin members. If there exist more than 1 kin members in neighborhood, then stay, else randomly move to one of eight neighboring empty places.

Collective Strategy with Move (CSM3) Cooperate with kin members and defect against non-kin members. If there exist more than 2 kin members in neighborhood, then stay, else randomly move to one of eight neighboring empty places.

Collective Strategy with Move (CSM4) Cooperate with kin members and defect against non-kin members. If there exist more than 3 kin members in neighborhood, then stay, else randomly move to one of eight neighboring empty places. 


\section{References}

1. Ahmed, S.A., Delfin S, G., Dirk, W.: Global migration revisited: short-term pains, long-term gains, and the potential of south-south migration. The World Bank (2016)

2. Van den Berg, H., Bodvarsson, Ö.B.: The Economics of Immigration: Theory and Policy. Springer (2009)

3. Boyd, R., Richerson, P.J.: The evolution of reciprocity in sizable groups. Journal of theoretical Biology 132(3), 337-356 (1988)

4. Card, D., Dustmann, C., Preston, I.: Immigration, wages, and compositional amenities. Journal of the European Economic Association 10(1), 78-119 (2012)

5. Chiong, R., Kirley, M.: Random mobility and the evolution of cooperation in spatial n-player iterated prisoner's dilemma games. Physica A: Statistical Mechanics and its Applications 391(15), 3915-3923 (2012)

6. Di Giovanni, J., Levchenko, A.A., Ortega, F.: A global view of cross-border migration. Journal of the European Economic Association 13(1), 168-202 (2015)

7. Gordon, D.M.: The ecology of collective behavior. PLoS Biology 12(3) (2014)

8. Hamilton, W.D.: The genetical evolution of social behaviour. (ii). Journal of theoretical biology $\mathbf{7}(1), 17-52$ (1964)

9. Herbert, B.: New outline of the principles of sociology. NY: Barnes and Nobel p. $67121(1951)$

10. Liebrand, W.B.: A classification of social dilemma games. Simulation \& Games 14(2), 123-138 (1983). https://doi.org/10.1177/104687818301400201

11. London, E.R.C.: Who's who: Definitions (9 2015), an optional note

12. Meloni, S., Buscarino, A., Fortuna, L., Frasca, M., Gómez-Gardeñes, J., Latora, V., Moreno, Y.: Effects of mobility in a population of prisoner's dilemma players. Physical Review E 79(6), 067101 (2009)

13. Nowak, M.A.: Evolutionary dynamics. Harvard University Press (2006)

14. Park, R., Burgess, E.: Introduction to the science of sociology. The University of Chicago (1921)

15. Rapoport, A.: Two-person game theory. Courier Corporation (2013)

16. Ren, Y., Chen, X., Wang, Z., Shi, B., Cui, G., Wu, T., Choo, K.K.R.: Neighborconsidered migration facilitates cooperation in prisoner's dilemma games. Applied Mathematics and Computation 323, 95-105 (2018)

17. Rogers, T., McKane, A.J.: A unified framework for schelling's model of segregation. Journal of Statistical Mechanics: Theory and Experiment 2011(07), P07006 (2011)

18. Santos, F.C., Pacheco, J.M.: Scale-free networks provide a unifying framework for the emergence of cooperation. Physical Review Letters 95(9), 098104 (2005)

19. Smith, J.M.: Evolution and the Theory of Games. Cambridge university press (1982)

20. Smith, J.M., Szathmary, E.: The major transitions in evolution. Oxford University Press (1997)

21. Suzuki, S., Akiyama, E.: Reputation and the evolution of cooperation in sizable groups. Proceedings of the Royal Society B: Biological Sciences 272(1570), 13731377 (2005)

22. Szabó, G., Fath, G.: Evolutionary games on graphs. Physics reports 446(4-6), 97$216(2007)$

23. Vainstein, M.H., Silva, A.T., Arenzon, J.J.: Does mobility decrease cooperation? Journal of theoretical biology 244(4), 722-728 (2007) 